www.nature.com/jhg

\title{
Haplotype analysis of ESR2 in Japanese patients with spermatogenic failure
}

\author{
Tsutomu Ogata ${ }^{1,2}$, Maki Fukami $^{2}$, Rie Yoshida ${ }^{2}$, Eiko Nagata $^{1}$, Yasuko Fujisawa ${ }^{1}$, Atsumi Yoshida ${ }^{3}$ and \\ Yasunori Yoshimura ${ }^{4}$
}

The prevalence of spermatogenic failure (SF) has gradually increased during the past few decades at least in several countries. Although multiple factors would be involved in this phenomenon, one important factor would be excessive estrogen effects via estrogen receptors (ERs). Thus, we performed haplotype analysis of ESR2 encoding ER $\beta$ in 125 Japanese SF patients and 119 age-matched control males, using single nucleotide polymorphisms (SNPs) 1-9 that are widely distributed on the $\sim 120-\mathrm{kb}$ genomic sequence of ESR2. Consequently, a linkage disequilibrium (LD) block was detected in an $\sim 60$-kb region encompassing SNPs 2-7 in both groups, and four major estimated haplotypes were identified within the LD block. Furthermore, the most prevalent 'TGTAGA' haplotype was found to be significantly associated with SF, with the $P$-value obtained by the CochranArmitage trend test $(0.0029)$ being lower than that obtained by a 100000 -times permutation test $(0.0038)$ to cope with the problem of multiple comparisons. The results, in conjunction with our previous data indicating lack of a susceptibility factor on ESR1 encoding ER $\alpha$, imply that the specific 'TGTAGA' haplotype of ESR2 raises the susceptibility to the development of SF. Journal of Human Genetics (2012) 57, 449-452; doi:10.1038/jhg.2012.53; published online 24 May 2012

Keywords: environmental endocrine disruptors; ESR2; estrogenic effects; haplotype analysis; spermatogenic failure; susceptibility

\section{INTRODUCTION}

Recent studies have indicated a gradual increase in the prevalence of male genial and reproductive abnormalities during the past few decades at least in several countries. ${ }^{1}$ Skakkebaek et al. ${ }^{2}$ have coined a term 'testicular dysgenesis syndrome' for this phenomenon. As such deterioration of male genital and reproductive health is also observed in many wildlife species, ${ }^{1,3}$ it is likely that such adverse changes in males are inter-related events shared in common by the human and the wildlife species. ${ }^{1,3}$ In this regard, environmental endocrine disruptors (EEDs) appear to constitute the major factor for this phenomenon, because EEDs are widely spread in the world. ${ }^{1,3}$ In particular, exposure to estrogenic EEDs are known to affect male genital and reproductive health. ${ }^{1,3-5}$

The effects of EEDs would primarily be determined by the genetic susceptibility, together with the dosage of exposed EEDs, character of exposed EEDs (for example, estrogenic, anti-androgenic and so on), and the developmental stage of the individuals at the time of EED exposure. ${ }^{1,3}$ In this regard, it is known that estrogenic EEDs can bind to both estrogen receptor (ER) $\alpha$ encoded by ESR 1 and ER $\beta$ encoded by ESR2 with low but variable degrees of affinities. ${ }^{3}$ Thus, it is likely that genetic susceptibility to estrogenic EEDs is primarily constituted by genetic variations in ESR1 and ESR2. 1,3

To examine this possibility, we have previously performed haplotype analysis of ESR1 in Japanese male patients with genial and reproductive abnormalities as well as in control males, using 15 single nucleotide polymorphisms (SNPs 1-15) that are widely distributed throughout the $>300-\mathrm{kb}$ genomic sequence of ESR1.6,7 Consequently, we identified an $\sim 50-\mathrm{kb}$ linkage disequilibrium (LD) block spanning SNPs $10-14$ in the $3^{\prime}$ region of ESR1, and found that homozygosity of a specific 'AGATA' haplotype within the LD block was strongly associated with cryptorchidism $(P=0.0040$; odds ratio $(\mathrm{OR})=7.55)$ and hypospadias $(P=0.000057 ; \mathrm{OR}=13.75)^{6,7}$ (and our unpublished updated observation). This finding provides strong evidence that homozygosity of the specific ESR1 haplotype raises the susceptibility to the development of male genital abnormalities. In this context, we speculate that this effect via the specific ESR1 haplotype is mediated by EEDs, although there is no direct evidence yet. Indeed, as ESR1 is expressed in Leydig cells producing testosterone and insulin-like $3,5,8$ it is likely that the specific ESR1 haplotype primarily enhances estrogenic effects in Leydig cells, compromising their hormonal production capacity.

However, no significant association was found between the specific 'AGATA' haplotype of ESR1 and spermatogenic failure (SF). ${ }^{7}$ In this context, as ESR2 is clearly expressed in various developmental stages of male germ cells, ${ }^{5}$ it may be possible that the deleterious effects of estrogenic EEDs on spermatogenesis may primarily be mediated by ER $\beta$. Thus, we carried out haplotype analysis of ESR2 in Japanese patients with SF.

${ }^{1}$ Department of Pediatrics, Hamamatsu University School of Medicine, Hamamatsu, Japan; ${ }^{2}$ Department of Molecular Endocrinology, National Research Institute for Child Health and Development, Tokyo, Japan; ${ }^{3}$ Kiba Park Clinic, Tokyo, Japan and ${ }^{4}$ Department of Obstetrics and Gynecology, Keio University School of Medicine, Tokyo, Japan

Correspondence: Professor T Ogata, Department of Pediatrics, Hamamatsu University School of Medicine, 1-20-1, Handayama, Higashi-ku, Hamamatsu, Shizuoka 431-3192, Japan.

E-mail: tomogata@hama-med.ac.jp

Received 28 November 2011; revised 25 April 2012; accepted 28 April 2012; published online 24 May 2012 


\section{MATERIALS AND METHODS}

\section{Subjects}

We studied 125 SF patients aged 32-52 years (median 41.0 years), including 80 SF patients utilized in the previous ESR1 haplotype analysis. ${ }^{7}$ The selection criteria included: (1) azoospermia or severe oligozoospermia ( $<5$ million sperms per $\mathrm{ml}$ ) demonstrated by two consecutive analyses of semen obtained after 4-7 days of abstinence; (2) lack of extragenital anomalies such as cryptorchidism and hypospadias; (3) hypergonadotropic hypogonadism indicative of primary testicular dysfunction; (4) no seminal tract obstruction, varicocele, or retrograde ejaculation; (5) a 46,XY karyotype with no demonstrable structural or numerical abnormality after examining $\geqslant 30$ lymphocytes; (6) absence of a Y chromosomal microdeletion after examining 36 loci from $S R Y$ to $D Y Z 1$, including multiple Yq loci in the azoospermia factor regions (AZFa, b, c) such as $R B M Y$ and $D A Z ;^{9}$ (7) no significant expansion of CAG repeat length at exon 1 of $A R$ that is known to raise the susceptibility to male reproductive abnormalities; ${ }^{10}$ and (8) lack of a disease episode that could affect fertility such as mumps orchiditis. For controls, 119 control adult males with proven fertility aged 24-50years (median 35.5 year) were similarly analyzed with permission. The ages were similar between the SF patients and control males (Mann-Whitney's $U$-test). All the SF patients and control males were Japanese living in the Tokyo urban area; they were free from particular residential environments such as the vicinity of chemical factories or farms, from specific dietary habits deviated to vegetables or animal/fish proteins, and from intake of drugs with hormonal effects.

\section{SNP analysis}

This study was approved by the Institutional Review Board Committees of the authors, and informed consent was obtained from each subject. We examined nine SNPs (SNPs 1-9) that were associated with high minor allele frequencies in the Japanese population (20.3-39.5\%) (the NCBI Short Genetic Variations Database (dbSNP); http://www.ncbi.nlm.nih.gov/snp/) and were widely distributed on the $\sim 120-\mathrm{kb}$ ESR2 genomic DNA sequence including an apparent LD block encompassing exons 1-6 identified in various populations (the International HapMap Project Database; http://hapmap.ncbi.nlm.nih.gov/) (Figure 1a). Genotyping was performed by the $5^{\prime}$ nuclease assay on an ABI PRISM 7000 Sequence Detection System (Life Technologies, Carlsbad, CA, USA), ${ }^{11}$ using leukocyte genomic DNA of each subject.

Pearson's $\chi^{2}$-test with one degree of freedom was applied to test whether the genotyping data are in the Hardy-Weinberg equilibrium. Statistical significance of the differences in allele and genotype frequencies was analyzed by
Pearson's $\chi^{2}$-test, using R environment for statistical computing (http://www.rproject.org/).

\section{Haplotype analysis}

Although haplotypes are usually not observed, the haplotypes present in a subject and the frequencies of the haplotypes in a population can be inferred using genotype data at separate loci. ${ }^{12}$ In this regard, the degree of LD can be expressed as the pairwise $\left|D^{\prime}\right|$ value (the absolute value for the disequilibrium parameter) that ranges from 0 (complete absence of LD) to 1.0 (complete presence of $\mathrm{LD}$ ), ${ }^{13}$ and a chromosomal region associated with high $\left|D^{\prime}\right|$ values between different loci is defined as a haplotype or an LD block. ${ }^{14}$ In this study, haplotype inference was performed by the maximum-likelihood method using expectation maximization algorithm implemented in the software LDSUPPORT. ${ }^{15,16}$ The pairwise $\left|D^{\prime}\right|$ values were estimated by the method of Terwilliger and Ott, ${ }^{12}$ and a haplotype block was determined by the method of Zhu et al. ${ }^{17}$ using the software developed by Kamatani et al. ${ }^{18}$

The difference in the frequencies of haplotypes between the SF patients and the control males was examined using the estimated population haplotype frequencies by Pearson's $\chi^{2}$-test, and the OR and the $95 \%$ confidence interval (CI) were calculated using the $\mathrm{R}$ environment. The association between $\mathrm{SF}$ phenotype and estimated haplotypes was tested using PENHAPLO software in a dominant mode (comparison of the frequencies of subjects with one risk haplotype between cases and controls) and in a recessive mode (comparison of the frequencies of subjects with two risk haplotypes between cases and controls). ${ }^{19}$ Furthermore, the association between SF phenotype and estimated haplotypes was also examined in a dosage-dependent mode (comparison of the frequencies of subjects with zero, one, and two risk haplotypes between cases and controls) by the Cochran-Armitage trend test, ${ }^{20,21}$ using the $\mathrm{R}$ environment. To cope with the problem of multiple comparisons, the significant level was determined by a 100000 -times permutation test. ${ }^{22}$

\section{RESULTS}

\section{SNP analysis}

The results of SNP analysis are summarized in Table 1. Minor allele frequencies of the 9 SNPs were $20.4-46.8 \%$ in the SF patients and $27.7-37.3 \%$ in control males. The genotype frequencies of SNPs $1-9$ were in accord with the Hardy-Weinberg equilibrium. Low $P$-values $(<0.05)$ were identified for the differences in the allele and genotype frequencies of SNPs 1, 4, and 5, with stronger association being identified for the

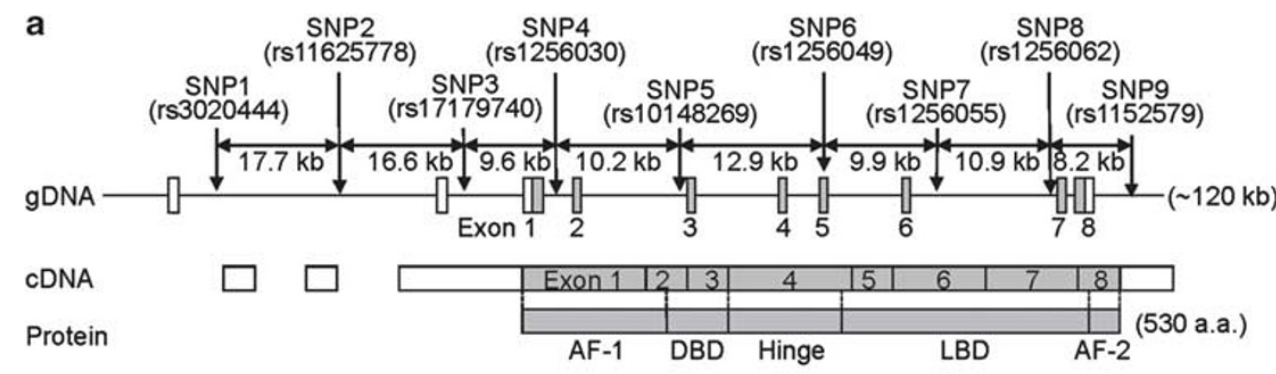

b

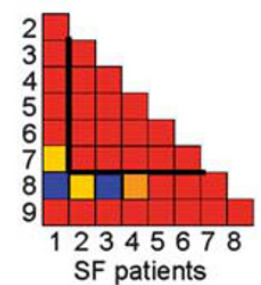

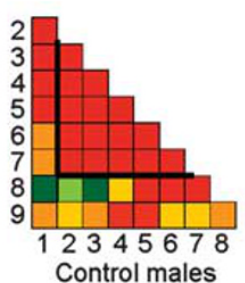

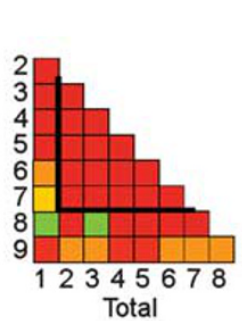

|D'|

$0-0.1$

$0.2-0.3$

$0.3-0.4$

$0.4-0.5$

$0.5-0.6$

$0.6-0.7$

$0.7-0.8$

$0.9-1.0$

Figure 1 Schematic representation of ESR2 and its LD maps. (a) Physical positions of ESR2 SNPS 1-9 examined in the present study. The gray and the white boxes represent coding and untranslated regions, respectively. AF-1, activation function 1 (ligand independent); AF-2: activation function 2 (ligand dependent); DBD, DNA-binding domain; LBD, ligand-binding domain. (b) Pairwise LD maps. $|D|$ : an absolute value for the disequilibrium parameter. 
Table 1 Summary of SNP analysis

\begin{tabular}{|c|c|c|c|c|c|c|c|}
\hline \multicolumn{4}{|c|}{ Genotyping data } & \multicolumn{4}{|c|}{ Statistical data } \\
\hline & Genotype & $S F$ & $C M$ & & $P$-value & $O R$ & $95 \% \mathrm{Cl}$ \\
\hline SNP1 & $\mathrm{TT}$ & 78 & 58 & T vs $C$ & 0.028 & 1.59 & $1.05-2.42$ \\
\hline \multirow[t]{2}{*}{ rs3020444 } & TC & 43 & 53 & TT vs TC $+\mathrm{CC}$ & 0.032 & 0.57 & $0.34-0.95$ \\
\hline & $\mathrm{CC}$ & 4 & 8 & $\mathrm{TT}+\mathrm{TC}$ vs CC & 0.20 & 2.18 & $0.64-7.44$ \\
\hline SNP2 & TT & 68 & 63 & T vs $C$ & 0.74 & 1.07 & $0.72-1.56$ \\
\hline \multirow[t]{2}{*}{ rs11625778 } & $\mathrm{TC}$ & 48 & 46 & $\mathrm{TT}$ vs $\mathrm{TC}+\mathrm{CC}$ & 0.82 & 0.94 & $0.57-1.56$ \\
\hline & $\mathrm{CC}$ & 9 & 10 & $\mathrm{TT}+\mathrm{TC}$ vs $\mathrm{CC}$ & 0.73 & 1.18 & 0.4 \\
\hline SNP3 & GG & 77 & 59 & $G$ vs $A$ & 0.059 & 1.49 & $0.98-2.25$ \\
\hline \multirow[t]{2}{*}{ rs17179740 } & AG & 43 & 52 & $G G$ vs $A G+A A$ & 0.059 & 0.61 & 1.02 \\
\hline & $\mathrm{AA}$ & 5 & 8 & $\mathrm{GG}+$ & 0.34 & 1.73 & \\
\hline SNP4 & $\mathrm{CC}$ & 36 & 55 & $C$ vs T & 0.0022 & 1.77 & $1.23-2.56$ \\
\hline \multirow[t]{2}{*}{ rs 1256030} & СT & 61 & 49 & $\mathrm{CC}$ vs $\mathrm{CT}+\mathrm{TT}$ & 0.0 & 2.13 & -3.61 \\
\hline & TT & 28 & 15 & $\mathrm{CC}+\mathrm{CT}$ & 0.0 & 0.500 & 0.25 \\
\hline SNP5 & GG & 36 & 55 & $G$ vs $A$ & 0.0022 & 1.77 & 1.2 \\
\hline \multirow[t]{2}{*}{ rs10148269 } & $A G$ & 61 & 49 & $G G$ vs $A G+A A$ & 0.0049 & 2.13 & $1.25-3.61$ \\
\hline & $\mathrm{AA}$ & 28 & 15 & $G G+A G$ vs $A A$ & 0.045 & 0.500 & $0.25-0.99$ \\
\hline SNP6 & GG & 68 & 64 & $G$ vs $A$ & 0.74 & 1.07 & $0.72-1.60$ \\
\hline \multirow[t]{2}{*}{ rs1256049 } & GA & 49 & 45 & $G G$ vs $G A+A A$ & 0.92 & 0.98 & $0.59-1.61$ \\
\hline & $\mathrm{AA}$ & 8 & 10 & $\mathrm{GG}+\mathrm{GA}$ vs $\mathrm{AA}$ & 0.55 & 1.34 & $0.51-3.52$ \\
\hline SNP7 & $\mathrm{AA}$ & 68 & 64 & $A$ vs $G$ & 0.74 & 1.07 & $0.72-1.60$ \\
\hline \multirow[t]{2}{*}{ rs1256055 } & $A G$ & 49 & 45 & $A A$ vs $A G+G G$ & 0.92 & 0.98 & $0.59-1.61$ \\
\hline & GG & 8 & 10 & $A A+A G$ vs $G G$ & 0.55 & 1.34 & $0.51-3.52$ \\
\hline SNP8 & $A A$ & 59 & 47 & $A$ vs $G$ & 0.21 & 1.27 & $0.87-1.85$ \\
\hline \multirow[t]{2}{*}{ rs1256062 } & $A G$ & 54 & 57 & $A A$ vs $A G+G G$ & 0.22 & 0.73 & $0.44-1.21$ \\
\hline & GG & 12 & 15 & $A A+A G$ vs $G G$ & 0.45 & 1.36 & $0.61-3.04$ \\
\hline SNP9 & GG & 40 & 45 & G vs $A$ & 0.12 & 1.34 & $0.93-1.92$ \\
\hline \multirow[t]{2}{*}{ rs1152579 } & GA & 59 & 59 & $\mathrm{GG}$ vs $\mathrm{GA}+\mathrm{AA}$ & 0.34 & 1.29 & $0.76-2.19$ \\
\hline & $A A$ & 26 & 15 & $\mathrm{GG}+\mathrm{GA}$ vs $\mathrm{AA}$ & 0.087 & 0.55 & $0.28-1.10$ \\
\hline
\end{tabular}

Abbreviations: $\mathrm{Cl}$, confidence interval; OR, odds ratio; SNP, single nucleotide polymorphism. NCBI rs no. is given for each SNP. SF, 125 patients with spermatogenic failure; CM, 119 control males.

allele rather than the genotype frequencies. In particular, the $P$-values for allele frequencies of SNPs 4 and 5 were markedly low.

\section{Haplotype analysis}

The LD map is shown in Figure 1b, and the results of haplotype analysis are summarized in Table 2 . An $\sim 60-\mathrm{kb}$ LD block spanning SNPs 2-7 was identified in both the SF patients and control males, with the $\left|D^{\prime}\right|$ value being $>0.9$ for all the pairs of SNPs $2-7$. Within the LD block, four major estimated haplotypes were identified, together with three additional minor haplotypes ('CGTAGA' haplotype in a single control male, and 'TATAGA' and 'CGCGGA' haplotypes in single SF patients). Notably, the frequency of the most prevalent 'TGTAGA' haplotype was significantly higher in the SF patients than in the control males. Furthermore, the 'TGTAGA' haplotype was significantly associated with SF phenotype, with the $P$-value obtained by the Cochran-Armitage trend test (0.0029) being lower than the permutation $P$-value (0.0038). In addition, of the four major haplotypes, the 'TGTAGA' haplotype alone contained the ' $\mathrm{T}$ ' allele in SNP 4 and the 'A' allele in SNP 5, whereas these two alleles were also identified in two of the three minor haplotypes.

\section{DISCUSSION}

The present study revealed the presence of an $\sim 60-\mathrm{kb}$ LD block encompassing SNPs 2-7 of ESR2 in both the SF patients and control males. In this regard, the allele frequencies obtained in the control males are comparable to those registered in the JSNP Database, and the LD
Table 2 Summary of haplotype analysis (SNPs 2-7)

\begin{tabular}{lcccc}
\hline Estimated haplotype & TGTAGA & TACGGA & CGCGAG & TGCGGA \\
\hline SF $(n=125)$ & $46.4 \%$ & $21.2 \%$ & $26.0 \%$ & $6.0 \%$ \\
CM $(n=119)$ & $32.7 \%$ & $28.1 \%$ & $27.3 \%$ & $11.0 \%$ \\
Comparison of estimated haplotype frequency & & \\
$\quad P$-value & 0.0028 & 0.096 & 0.82 & 0.070 \\
OR & 1.77 & 0.69 & 0.94 & 0.52 \\
$95 \% \mathrm{Cl}$ & $1.21-2.61$ & $0.44-1.06$ & $0.61-1.43$ & $0.25-1.05$
\end{tabular}

Association of estimated haplotype with phenotype

Dominant mode

$\begin{array}{ccccc}P \text {-value } & 0.0063 & 0.078 & 0.92 & 0.031 \\ \text { OR } & 2.08 & 0.63 & 0.98 & 0.46 \\ 95 \% \mathrm{Cl} & 1.23-3.54 & 0.38-1.05 & 0.59-1.62 & 0.22-0.93 \\ \begin{array}{c}\text { Recessive mode } \\ P \text {-value }\end{array} & 0.026 & 0.34 & 0.55 & 0.97 \\ \text { OR } & 2.16 & 0.58 & 0.75 & 0.95 \\ 95 \% \mathrm{Cl} & 1.09-4.46 & 0.17-1.79 & 0.28-1.96 & 0.037-24.2\end{array}$

Cochran-Armitage's trend test

$\begin{array}{lllll}P \text {-value } & 0.0029 & 0.071 & 0.75 & 0.056\end{array}$

For one haplotype

$\begin{array}{lllll}\text { OR } & 1.75 & 0.67 & 0.94 & 0.52\end{array}$

$95 \% \mathrm{Cl} \quad 1.21-2.52 \quad 0.44-1.03 \quad 0.63-1.39 \quad 0.27-1.02$

For two haplotypes

$\begin{array}{lcccc}\text { OR } & 3.06 & 0.45 & 0.88 & 0.27 \\ 95 \% \mathrm{Cl} & 1.46-6.35 & 0.19-1.06 & 0.39-1.93 & 0.07-1.04\end{array}$

Abbreviations: $\mathrm{Cl}$, confidence interval; OR, odds ratio; SNP, single nucleotide polymorphism. SF, 125 patients with spermatogenic failure; CM, 119 control males.

block identified in this study is similar to that reported in the International HapMap Project. These findings argue for the accuracy of our data.

Of the four major estimated haplotypes within the LD block, the 'TGTAGA' haplotype was significantly associated with SF. Indeed, the $P$-value obtained by the Cochran-Armitage trend test was below the permutation $P$-value. Furthermore, comparison of the $P$-values obtained from the three types of analyses for the association between SF phenotype and estimated haplotypes implies that the specific 'TGTAGA' haplotype compromises spermatogenesis in a dosagedependent manner rather than in a simple dominant or recessive manner. In this regard, as the ' $T$ ' allele of SNP 4 and the ' $A$ ' allele of SNP 5 are almost exclusively present in the 'TGTAGA' haplotype, genotyping of SNPs 4 and 5 can be utilized for the screening of the 'TGTAGA' haplotype.

For ESR2, previous studies have suggested an association between SF and an RsaI SNP on exon 5 that does not result in amino acid change (SNP 6 in this study) in Scandinavian and Iranian populations ( $P$-value: 0.01 and 0.012 , respectively). ${ }^{23,24}$ In such studies, as the frequency of AG genotype relative to GG genotype was higher in SF patients than in control males (AA genotype was extremely rare), this would imply that the 'A' allele of SNP 6 is regarded as a marker for a hidden true susceptibility factor(s) that is probably in an LD status with the 'A' allele of SNP 6. By contrast, the present study showed no association of SF with SNP 6 and rather suggests a dosage effect of the specific haplotype harboring the ' $G$ ' allele of SNP 6. Thus, the present data are apparently inconsistent with the previous studies. It might be possible, however, that the true susceptibility factor(s) is linked with the specific 'TGTAGA' haplotype in the Japanese population and resides on a different pattern of haplotype carrying the 'A' allele of SNP 6 in Scandinavian and Iranian populations, because of a recombination between the true susceptibility factor(s) and SNP 6 in either of the ethnic groups. In addition, there might be population- 
specific susceptibility factors, and false positive results might be obtained in association studies with multiple comparisons. This matter awaits further studies.

One may argue that although the present study indicates an association of the specific ESR2 haplotype with SF, there is no direct evidence for estrogenic EEDs being involved in the development of SF. Indeed, it may be possible that an interaction between the specific ESR2 haplotype and endogenous estrogens rather than estrogenic EEDs actually underlie the development of SF. However, estrogenic effects of EEDs are known to be primarily mediated by ER. ${ }^{1,3}$ In addition, as all the SF patients and the control males examined in this study were apparently free from high exposure to EEDs, the amount of exposed EEDs would be similar between the two groups of subjects. Thus, although further studies such as the investigation of subjects with a high risk of EEDs exposure (for example, workers at chemical factories) are necessary, our results would suggest that the specific ESR2 haplotype constitutes a susceptibility factor for the development of SF in response to estrogenic EEDs in males who live in an ordinary condition with no high risk of EEDs exposure.

Several points should be made with respect to the present study. First, the number of subjects analyzed remains rather small. Second, the true susceptibility factor(s) on the specific haplotype remains to be identified, although the specific 'TGTAGA' haplotype would facilitate the development of SF by enhancing the ER $\beta$ signaling. Third, it remains possible that another susceptibility factor(s) is present on ESR2. In particular, as only a few of SNPs were examined in non-LD block regions, a different susceptibility factor(s) may be present on the non-LD block regions of ESR2. Fourth, several patients may have some unidentified pathologic cause(s) for SF such as single gene disorders. Fifth, there may be some unknown minor genetic and environmental differences between the patients and the control males. In this context, as SF becomes discernible in adulthood, such minor differences, if they exist, may exert unfavorable influences on spermatogenic function for a long time, leading to SF. This may explain why the OR obtained in this study remained low, in contrast to the high ORs identified in cryptorchidism (7.55) and hypospadias $(13.75)^{6,7}$ (and our unpublished updated observation) which develop during the fetal life. Sixth, although it is known that EEDs also exert anti-androgenic effects and influence aromatization, ${ }^{25,26}$ these have not been examined in this study. Lastly, it remains to be determined whether similar results can be reproduced in other case-control studies.

Despite the above caveats, this study provides a useful clue to clarify the genetic susceptibility to estrogenic EEDs. In summary, we propose that the specific ESR2 haplotype raises the susceptibility to the development of SF in response to estrogenic EEDs. Further studies including similar haplotype analyses in different ethnic groups from both developed and developing countries will serve to clarify the relative importance of the dosage of exposed EEDs and the genetic heterogeneity obtained in the process of natural human selection, in the presumably EEDs-related phenomenon such as SF.

\section{CONFLICT OF INTEREST}

The authors declare no conflict of interest.

\section{ACKNOWLEDGEMENTS}

We would like to thank Dr Kamitsuji at StaGen Co., Ltd. for his critical advice in the genetic statistical analyses. This study was supported in part by the Environment Research and Technology Development Fund (C-0905) of the Ministry of Environment, by the Grants for Research on Intractable Diseases (H22-098), Health Research on Children, Youth and Families (H21-005), and Research on Risk of Chemical Materials (H20-004) from the Ministry of
Health, Labor and Welfare, by the Grants-in-Aid for Scientific Research (S) (22227002) from the Japan Society for the Promotion of Science (JSPS), and Grant-in-Aid for Scientific Research on Innovative Areas (22132004) from the Ministry of Education, Culture, Sports, Science and Technology (MEXT), and by the Grant from National Center for Child Health and Development (20C-2).

1 Toppari, J., Larsen, J. C., Christiansen, P., Giwercman, A., Grandjean, P., Guillette, Jr. L. J. et al. Male reproductive health and environmental xenoestrogens. Environ. Health Perspect. 104, (Suppl 4) 741-803 (1996).

2 Skakkebaek, N. E., Rajpert-De Meyts, E. \& Main, K. M. Testicular dysgenesis syndrome: an increasingly common developmental disorder with environmenta aspects. Hum. Reprod. 16, 972-978 (2001).

3 McLachlan, J. A. Environmental signaling. what embryos and evolution teach us about endocrine disrupting chemicals. Endocr. Rev. 22, 319-341 (2001).

4 Stillman, R. J. In utero exposure to diethylstilbestrol: adverse effects on the reproductive tract and reproductive performance and male and female offspring. Am. J. Obstet. Gynecol. 142, 905-921 (1982).

5 O'Donnell, L., Robertson, K. M., Jones, M. E. \& Simpson, E. R. Estrogen and spermatogenesis. Endocr. Rev. 22, 289-318 (2001).

6 Yoshida, R., Fukami, M., Sasagawa, I., Hasegawa, T., Kamatani, N. \& Ogata, T. Association of cryptorchidism with a specific haplotype of the estrogen receptor alpha gene: implication for the susceptibility to estrogenic environmental endocrine disruptors. J. Clin. Endocrinol. Metab. 90, 4716-4721 (2005).

7 Watanabe, M., Yoshida, R., Ueoka, K., Aoki, K., Sasagawa, I., Hasegawa, T. et al. Haplotype analysis of the estrogen receptor 1 gene in male genital and reproductive abnormalities. Hum. Reprod. 22, 1279-1284 (2007).

8 Foresta, C., Zuccarello, D., Garolla, A. \& Ferlin, A. Role of hormones, genes, and environment in human cryptorchidism. Endocr. Rev. 29, 560-580 (2008).

9 Vogt, P. H. AZF deletions and Y chromosomal haplogroups: history and update based on sequence. Hum. Reprod. Update 11, 319-336 (2005).

10 Dowsing, A. T., Yong, E. L., Clark, M., McLachlan, R. I., de Kretser, D. M. \& Trouson, A. O. Linkage between male infertility and trinucleotide repeat expansion in the androgen-receptor gene. Lancet 354, 640-643 (1999).

11 De La Vega, F. M., Dailey, D., Ziegle, J., Williams, J., Madden, D. \& Gilbert, D. A. New generation pharmacogenomic tools: a SNP linkage disequilibrium map, validated SNP assay resource, and high-throughput instrumentation system for large-scale genetic studies. Biotechniques 32, S48-S54 (2002)

12 Terwilliger, J. D. \& Ott, J. Handbook of Human Genetic Linkage (Johns Hopkins University Press, Baltimore, 1994).

13 Lewontin, R. C. The interaction of selection and linkage. I. General considerations: heterotic models. Genetics 49, 49-67 (1964).

14 Kruglyak, L. Prospects for whole-genome linkage disequilibrium mapping of common disease genes. Nat. Genet. 22, 139-144 (1999).

15 Excoffier, L. \& Slatkin, M. Maximum-likelihood estimation of molecular haplotype frequencies in a diploid population. Mol. Biol. Evol. 12, 921-927 (1995).

16 Kitamura, Y., Moriguchi, M., Kaneko, H., Morisaki, H., Morisaki, T., Toyama, K. et al. Determination of probability distribution of diplotype configuration (diplotype distribution) for each subject from genotypic data using the EM algorithm. Ann. Hum. Genet. 66, 183-193 (2002)

17 Zhu, X., Yan, D., Cooper, R. S., Luke, A., Ikeda, M. A., Chang, Y. P. et al. Linkage disequilibrium and haplotype diversity in the genes of the renin-angiotensin system: findings from the family blood pressure program. Genome Res. 13, 173-181 (2003)

18 Kamatani, N., Sekine, A., Kitamoto, T., lida, A., Saito, S., Kogame, A. et al. Large scale single-nucleotide polymorphism (SNP) and haplotype analyses, using dense SNP maps, of 199 drug-related genes in 752 subjects: the analysis of the association between uncommon SNPs within haplotype blocks and the haplotypes constructed with haplotype-tagging SNPs. Am. J. Hum. Genet 75, 190-203 (2004).

19 Ito, T., Inoue, E. \& Kamatani, N. Association test algorithm between a qualitative phenotype and a haplotype or haplotype set using simultaneous estimation of haplotype frequencies, diplotype configurations, and diplotype-based penetrances. Genetics 168, 2339-2348 (2004).

20 Cochran, W. G. Some methods for strengthening the common chi-square tests Biometrics 10, 417-451 (1954).

21 Armitage, P. Tests for Linear Trends in Proportions and Frequencies. Biometrics 11, 375-386 (1955)

22 Becker, T. \& Knapp, M. A powerful strategy to account for multiple testing in the context of haplotype analysis. Am. J. Hum. Genet 75, 561-570 (2004).

23 Aschim, E. L., Giwercman, A., Ståhl, O., Eberhard, J., Cwikiel, M., Nordenskjöld, A. et al. The Rsal polymorphism in the estrogen receptor-beta gene is associated with male infertility. J. Clin. Endocrinol. Metab. 90, 5343-5348 (2005).

24 Safarinejad, M. R., Shafiei, N. \& Safarinejad, S. Association of polymorphisms in the estrogen receptors alpha, and beta (ESR1, ESR2) with the occurrence of male infertility and semen parameters. J. Steroid Biochem. Mol. Biol 122, 193-203 (2010).

25 Svechnikov, K., Izzo, G., Landreh, L., Weisser, J. \& Soder, O. Endocrine disruptors and Leydig cell function. J. Biomed. Biotechnol 2010, 684504 (2010).

26 Whitehead, S. A. \& Rice, S. Endocrine-disrupting chemicals as modulators of sex steroid synthesis. Best Pract. Res. Clin. Endocrinol. Metab 20, 45-61 (2006). 